\title{
Reduction in Seed Cotton Yield Corresponding with Symptom Severity Grades of Cotton Leaf Curl Disease (CLCuD) in Upland Cotton (Gossypium hirsutum L.)
}

\author{
Satish Kumar Sain ${ }^{1}$, Dilip Monga', Man Mohan², Aman Sharma ${ }^{3}$ and Jagdish Beniwal ${ }^{2}$ \\ ${ }^{1}$ ICAR-Central Institute for Cotton Research, Regional Station, Sirsa (Haryana), India \\ ${ }^{2}$ Department of Plant Pathology, CCS HAU, Hisar (Haryana), India \\ ${ }^{3}$ Punjab Agricultural University, Regional Research Station, Faridkot (Punjab), India \\ *Corresponding author
}

\section{A B S T R A C T}

Cotton leaf curl disease is the major constraint in cotton production in the

\begin{tabular}{|l|}
\hline Ke y w o r d s \\
Cotton leaf curl \\
virus disease, \\
$\begin{array}{l}\text { Disease resistance, } \\
\text { Symptom severity } \\
\text { grades, Disease } \\
\text { reaction scale, } \\
\text { Cotton yield }\end{array}$ \\
\hline Article Info \\
\hline $\begin{array}{l}\text { Accepted: } \\
\text { 22 October 2020 } \\
\text { Available Online: } \\
\text { 10 November 2020 }\end{array}$ \\
\hline
\end{tabular}
Indian subcontinent. A field study (2012-2017) was conducted to assess seed cotton yield on Bt cotton cultivars analogous to the symptom rating scales (DRS) of CLCuD. The DRS is used to derive the percent disease intensity (PDI). Presently, percent disease index (PDI) for resistance screening ranges between 0.1 to $50 \%$, corresponding to the DRS 1 to 6 , which is lower than the mathematical midpoint value ranging between 8.4 to $91.8 \%$ and breeding material with good yield potential may be ignored during screening. The average yield loss corresponding to the DRS 1, 2, 3, 4,5 , and 6 was $15,28,38.6,49.1,59.1$ and $72.1 \%$. Hence, the newly proposed disease response scale would be much more justifiable and reliable for decision-making and determining the level of resistance or susceptibility in the genotypes, because the estimated severity may be much lower than the actual severity.

\section{Introduction}

Cotton regarded as "white gold" is one of the major cash crops in the economy of developing countries. It has a major impact on agriculture, industries, and the economy of the Indian subcontinent. It is used as raw material for indigenous textile and oil industries and exported to many countries as cotton lint and other products of the cotton value chain. In India, cotton was cultivated in 12.24 million ha area with the production of 36.1 million bales of $170 \mathrm{~kg}$ each during 2018-19 (Anonymous 2019). The north zone of India covers $12 \%$ of the cotton area and $17 \%$ production (Anonymous 2019). The 
demand and production of cotton have increased over the last decade, however, a lot of productivity is being reduced due to insectpests in general and CLCuD in particular in the northern cotton zone, which is the major hurdle in achieving the full potential of the varieties and the technologies. The viral agents associated with this disease are collectively known as cotton leaf curl virus disease (CLCuD) associated begomoviruses (CABs) in association with DNA beta and alpha satellites, which belong to the genus Begomovirus (family Geminiviridae), and are transmitted by the ubiquitous whitefly (Bemisia tabaci) (Mansoor et al., 2008; Brown et al., 2015, Sattar et al., 2017; Zerbini et al., 2017). CLCuD symptoms are characterized by upward or downward curling of leaves, darkening of veins, vein thickening, enations that appeared in a beard-shaped and small fine leaf-like structures on the undersurface of the leaves (Briddon et al., 2000, Briddon 2003; Kumar et al., 2010). Severe symptoms include stunted growth of the plants, reduced flowering, and boll formation. Production is adversely affected by CLCuD which leads to deterioration of fiber quality and yield (Farooq et al., 2011; Kumar et al., 2011; Singh et al., 2013).

The percent disease intensity (PDI) increases slowly during June and reaches up to $80 \%$ during August and September (Idris 1990; Ali et al., 1995; Monga et al., 1998). Monga et al., (2011) have recorded disease incidence 98\%, 95.4\% and 93.8 during 2001, 2000 and 2006, respectively in North India.

In Pakistan, the lint yield/ha dropped down by 29.3 and $36.5 \%$ percent, respectively, during 1992-1993 and 1993-1994 as compared to 1991-1992. Seed cotton yield reduction has also been reported in Rajasthan (32.9 to $50.3 \%$ ), Punjab (10.5 to $92.2 \%$ ) and Haryana (39.4 to $81.4 \%$ ) states of North India (Narula et al., 1999; Monga et al., 2001). CLCuD affects the yield and fiber quality of cotton crops posing a major threat to cotton cultivation in Northern India (Singh et al., 1994; Nateshan et al., 1996; Briddon 2003; Monga et al., 2004; Singh et al., 2013). Seed cotton yield loss estimation studies in Bt cotton hybrids from 2008-09 to 2010-11 based on PDI ranging from 5 to $60 \%$ and disease severity grades I- IV ranged from 0.08 to $59.5 \%$. Seed cotton yield reduction ranged from 7.2 to $80.1 \%$ at severity grades of one to four in different hybrids (Monga et al., 2011). Loss estimation studies due to CLCuD on $\mathrm{Bt}$ cotton hybrids in Punjab and Haryana during 2009-12 ranged between 25.2 and $46.6 \%$ (Monga et al., 2012). Seed cotton yield reduction ranging from 15.7 to $56.7 \%$ was recorded during 2012-13 and 2013-14 (Monga 2014). CLCuD was reported to cause average reduction in plant height (40.6\%), boll weight $(33.8 \%)$ and number of bolls plant $^{-1}(72.5-73.5 \%)$, GOT\% (3.93\%), seed index $(17.0 \%)$ and yield plant ${ }^{-1}(64.5 \%)$ (Ahmed 1999; Mahmood et al., 1996)

The variation in severity of CLCuD prevailing in the North Zone of India has been observed in farmer fields as well as research station screening trials. However, losses as per the symptom severity grades have not been properly defined. The symptom/disease rating scales (DRS) are used to derive the percent disease intensity (PDI). Work on screening of $\mathrm{CLCuD}$ resistance in cotton lines/cultivars is governed by the PDI corresponding to their level of resistance or susceptibility response/reaction. In the previous studies, the range of PDI which is used to decide the varieties/lines under highly resistant to the highly susceptible category also varies from the corresponding DRS and PDI. Moreover, the yield loss is in proportionate to the PDI may or may not occur, and the lines/germplasm with good yield potential may be ignored for future breeding improvement programmes. 
Considering the fact that the yield loss corresponding to CLCuD symptom severity grades/scale (DRS) which is used to arrive at PDI may vary, this study was conducted for assessment of seed cotton yield analogous to the symptom severity grades at three CLCuD hot spots fields in Haryana and Punjab from 2012 to 2017 on different Bt cotton cultivars.

\section{Materials and Methods}

\section{Experimental sites and period}

To conduct the study on the assessment of seed cotton yield analogous to the symptom severity grades, field experiments were conducted at two locations during six consecutive years from 2012-13 to 2017-18. The experiments were conducted at two selected sites (disease hot spots) under the All India Coordinated Research Programme on Cotton (AICRP on cotton) namely Agricultural Research Station, Punjab Agricultural University, Faridkot (Punjab) (30 40'33.3"N 7444'53.8"E), and CCS Haryana Agricultural University, Hisar (Haryana) $\left(29^{\circ} 09^{\prime} 16.9^{\prime \prime} \mathrm{N} \quad 75^{\circ} 41^{\prime} 51.7^{\prime \prime E}\right)$ as part of cooperative efforts of scientists under AICRP on cotton. These sites were selected based on the CLCUD severity recorded in the past under AICRP on the cotton programme. The Bt cotton (Gossypium hirsutum) hybrids were selected, among the cotton cultivars recommended by the Government of the respective states.

\section{Experimental design}

The field experiments were laid out in complete randomized block design (CRBD) with three replications at all the locations identified by AICRP on cotton for resistance screening against CLCuD. In each replication, the gross field plot size 250 to $500 \mathrm{~m}^{2}$ was maintained. The row to row and plant to plant spacing of $67.5 \times 60 \mathrm{~cm}$ was maintained and crops were cultivated as per the package of practices recommended by the respective State Agricultural Department/University. Any foliar chemical treatments were not applied for disease management except for the treatments applied for the planned experimental trial.

\section{Observation and data collection}

Data on CLCuD incidence were collected for estimating the percent disease incidence and disease intensity at 60,90 and 100 days after sowing. Observations of CLCuD on 50 cotton plants from each point/ location were recorded by observing CLCuD symptoms (Akthar et al., 2010; Monga, 2014). The observations were taken at 0-6 DRS (DRS) as described in Table 1.

The average grade was calculated by using the formula: Average disease rating grade = Sum of all disease rating grades/ Total number of plants, and percent disease intensity (PDI) was calculated for each entry/plot by using the following formula: $\mathrm{PDI}=$ (Average disease rating grades / Maximum disease rating grade) x 100 .

Similarly, data on the yield characteristics viz. the number of bolls per plant, seed cotton yield were recorded from 50 cotton plants falling under each DRS. The reduction of the number of bolls per plant and seed cotton yield was computed over the lowest grade recorded during the respective cropping season. Data on CLCuD were recorded using 0- 6 scale 2012 to 2017 at PAU, Faridkot, and from 2013-2017 at CCS HAU Hisar. The pooled data of both the location for each variety as well as for the respective grades were computed to draw the inference of the effect of CLCuD on the number of bolls, seed cotton yield under each DRS. Midpoint rule or the so-called trapezoidal integration method was used to evaluate the old disease 
response scale and to decide the proposed one (Campbell and Madden, 1990).

The observations were also taken for estimation of overall yield loss in each $\mathrm{Bt}$ hybrid during the experimental period. Disease severity of each Bt hybrid including plants with all types of symptom gradings was recorded. The CLCuD observations, number of bolls and seed cotton yield were also recorded from plants of each Bt hybrid grown in an area of 500 square meters from 2012 to 2017 at PAU, Faridkot, and from 2013-2017 at CCS HAU, Hisar.

Data were analyzed statistically and treatment effects were compared by Least Significant Difference Test $(L S D)$ at $0.05 \%$ with the help of the computer program SPSS software (IBM SPSS Statistics).

\section{Results and Discussion}

The study was conducted for the assessment of percent reduction in the number of bolls and seed cotton yield analogous to the symptom severity grades of cotton leaf curl virus disease (CLCuD) in upland cotton (Gossypium hirsutum) of North India. The data were collected during 2012- 2017 at CCS HAU, Hisar (Haryana) and during 2013-2017 at PAU, Faridkot (Punjab) on the number of bolls per plant and seed cotton yield (g/plant) corresponding to the CLCuD symptom severity grades/disease rating scale (DRS) in $\mathrm{Bt}$ cotton hybrids (BG-I, BG-II) recommended to the respective locations every year.

\section{Effect of CLCuD on number of bolls per plant}

The "0" DRS as described by Monga (2014) was observed only during 2012, 2016 and 2017 at PAU Faridkot and the highest number of bolls per plant under DRS "0" during these years was recorded in RCH-134 BG-I (116.2), Bioseed 6588 BG-II (54.4) and in Bioseed 6588 BG-II (58.3), respectively (Supplementary Table 1). During 2012, 2016 and 2017 the highest boll per plant corresponding to the DRS 0, 1, 2, 3, 4, 5 and 6 was recorded in RCH-134 BG-I (116.2), RCH-134 BG-I (93.2), RCH-134 BG-I (67.62), RCH-134 BG-I (51.4), Bioseed 6588 BG-II (42.0), and Bioseed 6588 (32.1). Similarly, during the year 2013, 2014, and 2015 DRS "0" was not recorded (Table 2).

The highest number of bolls per plant corresponding to the DRS 1, 2, 3, 4, 5 and 6 was recorded in RCH-134BG II (129.8), Bioseed 6588 (104.9), Bioseed 6588 (98.8), Ankur 3028 (77.2), RCH 650 (67.9), and Ankur 3028 (35.7) (LSD 8.317; $P \leq 0.05)$, respectively ( Supplementary Table 1).

The DRS "0" was observed only during 2013 in the Bt cotton hybrid RCH 134 BG-I and NCS55 BG-II at CCS HAU, Hisar, Haryana while in these two varieties DRS "1" was not observed. The DRS “0” from 2014 to 2017 , DRS 1 from 2015 to 2017 and DRS 2 during 2015 were not observed (Table 2).

The highest overall number of bolls per plant corresponding to the DRS $0,1,2,3,4,5$ and 6 was recorded in $\mathrm{RCH} 134$ (58.5), $\mathrm{RCH} 134$ (56.5), Bioseed 6588 (56.8), Ankur Jai (44.1), Ankur Jai Bt, BG-II (32.2), Ankur Jai 3028BG-II (27.8), and RCH 134 (15.3) (LSD 14.799; $\quad P \leq 0.05), \quad$ respectively (Supplementary Table 2).

The pooled range of reduction in the number of bolls per plant corresponding to the DRS 1, $2,3,4,5$ and 6 over the lowest DRS ranged from 1.5 to $32.2,5.1$ to $41.8,10.6$ to 60.2 , 25.7 to $65.1,33.6$ to 76.0 , and 33.3 to $97.2 \%$ (LSD 1.849-1.855; $P \leq 0.05$ ), respectively (Table 2). 


\section{Effect of CLCuD on seed cotton yield}

DRS "0" was observed only during 2012, 2016 and 2017 at ARS, Faridkot and the highest seed cotton per plant (g) corresponding to DRS $0,1,2,3,4,5$, and 6 was recorded in RCH BG-I (479.2), RCH BG-I (335.2), RCH BG-I (274.6), RCH BG-I (217.8), Bioseed 6588 (196.4), Ankur 3028 (179.5), and RCH 650 (149.4) ) (LSD 10.129; $P \leq 0.05$ ), respectively (Supplementary Table $3)$. Similarly, during the year 2013, 2014, and 2015 DRS "0" was not recorded. The highest seed cotton per plant $(\mathrm{g})$ in corresponding to DRS 1, 2, 3, 4, 5 and 6 was recorded in Ankur Jai Bt (356.2), Bioseed 6588 (314.7), Bioseed 6588 (296.4), Ankur 3028 (231.6), RCH 650 BG-II (203.7) and Ankur 3028 (107.1), respectively (Table 3 ). The highest seed cotton yield per plant $(\mathrm{g})$ in corresponding to DRS 0, 1, 2, 3, 4, 5 and 6 during 2013-17 at ARS, Hisar was observed in RCH 134 (205.65), RCH134 (173.91), RCH650 (223.54), RCH 650 (156.12), Ankur Jai
(110.63), Bioseed 6588 (125.71) and $\mathrm{RCH}$ 134 (58.65) (LSD 7.502; $P \leq 0.05)$, respectively ( Supplementary Table 3 ).

The pooled (\%) reduction in seed cotton yield per plant corresponding to DRS 1, 2, 3, 4, 5 and 6 was recorded in the range from 3.4 to $30.1,5.1$ to $46.1,10.6$ to $57.2,27.3$ to 69.3 , 37.3 to 83.3 and 23.2 to $97.4 \%$ (LSD 1.849$1.861 ; P \leq 0.05$ ), respectively (Table 3 ).

\section{An overall reduction in the number of bolls and seed cotton yield}

When comparing the overall reduction in the number of balls and seed cotton yield in each Bt hybrids during the experimental period, the highest seed cotton yield and number of bolls per plant were recorded during 2012 followed by 2017 . The lowest reduction was recorded during 2015 followed by 2014 (Table 2 and $3)$.

Table.1 Symptom and disease ratting scale proposed in the past (Akthar et al., 2010; Monga 2014)

\begin{tabular}{|c|c|c|c|c|}
\hline S.No. & Symptoms & $\begin{array}{l}\text { Disease rating } \\
\text { scale/ (DRS) } \\
\text { symptom } \\
\text { severity scale }\end{array}$ & $\begin{array}{l}\text { Range of } \\
\text { percent } \\
\text { disease } \\
\text { intensity }\end{array}$ & $\begin{array}{l}\text { Disease } \\
\text { response }\end{array}$ \\
\hline 1 & Complete absence of symptoms & 0 & 0 & Immune \\
\hline 2 & $\begin{array}{l}\text { Symptoms of vein thickening on a few } \\
\text { upper leaves }\end{array}$ & 1 & $0.1-10$ & $\begin{array}{l}\text { Highly } \\
\text { Resistant }\end{array}$ \\
\hline 3 & $\begin{array}{l}\text { Symptoms of vein thickening, cupping and } \\
\text { curling on few upper leaves }\end{array}$ & 2 & $11-20$ & Resistant \\
\hline 4 & $\begin{array}{l}25 \% \text { plant affected with vein thickening, } \\
\text { cupping, and curling, leaf enations }\end{array}$ & 3 & $21-30$ & $\begin{array}{l}\text { Moderately } \\
\text { Resistant }\end{array}$ \\
\hline 5 & $\begin{array}{l}50 \% \text { plant affected with vein thickening, } \\
\text { cupping, and curling, leaf enations }\end{array}$ & 4 & $31-40$ & $\begin{array}{l}\text { Moderately } \\
\text { Susceptible }\end{array}$ \\
\hline 6 & $\begin{array}{l}75 \% \text { plant affected } 5 \text { with vein thickening, } \\
\text { cupping, and curling, leafy enation }\end{array}$ & 5 & $41-50$ & Susceptible \\
\hline 7 & $\begin{array}{l}\text { Plants stunted severely and complete plant } \\
\text { affected with vein thickening, cupping and } \\
\text { curling, and leafy enation }\end{array}$ & 6 & $>51$ & $\begin{array}{c}\text { Highly } \\
\text { Susceptible }\end{array}$ \\
\hline
\end{tabular}


Table.2 Pooled reduction in the number of bolls per plant under different CLCuD grades (2012-2017)

\begin{tabular}{|c|c|c|c|c|c|c|c|c|c|c|c|c|c|c|c|c|c|c|c|c|c|c|c|c|}
\hline \multirow{3}{*}{$\begin{array}{c}\text { DRS } \\
\text { (grade) }\end{array}$} & \multicolumn{24}{|c|}{ Reduction in number of bolls per plant under different CLCuD grades (\%) } \\
\hline & \multicolumn{4}{|c|}{2012} & \multicolumn{4}{|c|}{2013} & \multicolumn{4}{|c|}{2014} & \multicolumn{4}{|c|}{2015} & \multicolumn{4}{|c|}{2016} & \multicolumn{4}{|c|}{2017} \\
\hline & $\begin{array}{l}\mathrm{RCH} \\
134\end{array}$ & $\begin{array}{l}\mathrm{RCH} \\
134\end{array}$ & $\begin{array}{l}\text { Ankur } \\
\text { Jai }\end{array}$ & $\begin{array}{l}\text { NCS } \\
855\end{array}$ & $\begin{array}{l}\mathrm{RCH} \\
134\end{array}$ & $\begin{array}{l}\mathrm{RCH} \\
134\end{array}$ & $\begin{array}{l}\text { Ankur } \\
\text { Jai }\end{array}$ & $\begin{array}{l}\text { NCS } \\
855\end{array}$ & $\begin{array}{l}\mathrm{RCH} \\
134\end{array}$ & $\begin{array}{l}\mathrm{RCH} \\
134\end{array}$ & $\begin{array}{l}\text { Ankur } \\
3028\end{array}$ & $\begin{array}{l}\text { NCS } \\
855\end{array}$ & $\begin{array}{l}\text { MRC } \\
7017\end{array}$ & $\begin{array}{l}\mathrm{RC} \\
\mathrm{H} \\
650\end{array}$ & $\begin{array}{l}\text { Ank } \\
\text { ur } \\
3028\end{array}$ & $\begin{array}{l}\text { Biose } \\
\text { ed } \\
6588\end{array}$ & $\begin{array}{l}\text { MRC } \\
7017\end{array}$ & $\begin{array}{l}\mathrm{RC} \\
\mathrm{H} \\
650\end{array}$ & $\begin{array}{l}\text { Ank } \\
\text { ur } \\
3028\end{array}$ & $\begin{array}{l}\text { Bios } \\
\text { eed } \\
6588\end{array}$ & $\begin{array}{l}\text { MR } \\
\text { C70 } \\
17\end{array}$ & $\begin{array}{l}\mathrm{RC} \\
\mathrm{H} \\
650\end{array}$ & $\begin{array}{l}\text { Ank } \\
\text { ur } \\
3028\end{array}$ & $\begin{array}{l}\text { Bios } \\
\text { eed } \\
6588\end{array}$ \\
\hline $\mathbf{0}$ & - & - & - & - & - & - & - & - & - & - & - & - & - & - & - & - & - & - & - & - & - & - & - & - \\
\hline 1 & 19.8 & 17.4 & 15.9 & 32.2 & - & - & - & - & - & - & - & - & - & - & - & - & 1.5 & 3.8 & 2.1 & 5.3 & 8.1 & 10.3 & 10.1 & 6.2 \\
\hline 2 & 41.8 & 43.0 & 36 & 28.1 & 21.6 & 24.4 & 5.1 & 17.9 & 8.1 & 20.0 & 13.8 & 15.5 & 12.9 & 6.3 & 12.0 & 5.1 & 5.3 & 9.2 & 12.1 & 6.4 & 9.4 & 15.7 & 17.4 & 13.4 \\
\hline 3 & 51.3 & 60.2 & 48.1 & 43.8 & 41.4 & 35.0 & 29.7 & 35.1 & 26.3 & 31.9 & 21.8 & 30.4 & 19.9 & 10.7 & 16.7 & 10.6 & 13.7 & 16.0 & 18.1 & 14.6 & 22.9 & 25.1 & 22.9 & 23.4 \\
\hline 4 & 55.8 & 65.1 & 62.0 & 51.8 & 61.1 & 52.9 & 44.0 & 51.7 & 36.3 & 39.2 & 47.7 & 44.3 & 25.7 & 27.0 & 31.3 & 36.5 & 28.0 & 29.2 & 30.1 & 24.5 & 35.3 & 39.8 & 31.1 & 41.4 \\
\hline 5 & 73.8 & 76.0 & 66.2 & 62.0 & 64.5 & 63.6 & 56.3 & 63.4 & 57.5 & 60.1 & 50.9 & 65.4 & 35.3 & 33.6 & 42.6 & 47.7 & 42.8 & 44.7 & 41.0 & 38.7 & 45.1 & 49.4 & 37.5 & 52.7 \\
\hline 6 & 89.7 & 86.4 & 79.2 & 82.1 & 79.4 & 79.2 & 73.4 & 71.4 & 83.8 & 97.2 & 95.3 & 93.9 & 60.3 & 52.5 & 64.4 & 76.2 & 45.9 & 48.2 & 43.3 & 41.0 & 60.6 & 65.4 & 48.0 & 65.4 \\
\hline $\begin{array}{c}\text { Over all } \\
\text { average } \\
\text { grade }\end{array}$ & 65.6 & 70.0 & 58.9 & 60.7 & 36.0 & 38.1 & 36.8 & 34.7 & 39.5 & 37.4 & 36.6 & 28.8 & 4.2 & 12.5 & 21.4 & 19.4 & 29.0 & 26.0 & 23.8 & 20.1 & 29.0 & 35.5 & 30.7 & 37.8 \\
\hline
\end{tabular}

Table.3 Pooled reduction in seed cotton yield per plant under different CLCuD grades (2012-2017)

\begin{tabular}{|c|c|c|c|c|c|c|c|c|c|c|c|c|c|c|c|c|c|c|c|c|c|c|c|c|}
\hline \multirow[t]{3}{*}{ DRS (grade) } & \multicolumn{24}{|c|}{ Reduction in Seed cotton yield under different CLCuD grades (\%) } \\
\hline & \multicolumn{4}{|c|}{2012} & \multicolumn{4}{|c|}{2013} & \multicolumn{4}{|c|}{2014} & \multicolumn{4}{|c|}{2015} & \multicolumn{4}{|c|}{2016} & \multicolumn{4}{|c|}{2017} \\
\hline & $\begin{array}{l}\mathrm{RC} \\
\mathrm{H} \\
134\end{array}$ & $\begin{array}{l}\mathrm{RC} \\
\mathrm{H} \\
134\end{array}$ & $\begin{array}{l}\text { Anku } \\
\text { r Jai }\end{array}$ & $\begin{array}{l}\text { NCS } \\
855\end{array}$ & $\begin{array}{l}\mathrm{RC} \\
\mathrm{H} \\
134\end{array}$ & $\begin{array}{l}\mathrm{RC} \\
\mathrm{H} \\
134\end{array}$ & $\begin{array}{l}\text { Ank } \\
\text { ur } \\
\text { Jai }\end{array}$ & $\begin{array}{l}\text { NCS } \\
855\end{array}$ & $\begin{array}{l}\mathrm{RC} \\
\mathrm{H} \\
134\end{array}$ & $\begin{array}{l}\mathrm{RC} \\
\mathrm{H} \\
134\end{array}$ & $\begin{array}{l}\text { Ank } \\
\text { ur } \\
3028\end{array}$ & $\begin{array}{l}\text { NCS } \\
855\end{array}$ & $\begin{array}{l}\text { MR } \\
\text { C70 } \\
17\end{array}$ & $\begin{array}{l}\mathrm{RCH} \\
650\end{array}$ & $\begin{array}{l}\text { Ank } \\
\text { ur } \\
3028\end{array}$ & $\begin{array}{l}\text { Bios } \\
\text { eed } \\
6588\end{array}$ & $\begin{array}{l}\text { MR } \\
\text { C70 } \\
17\end{array}$ & $\begin{array}{l}\mathrm{RC} \\
\mathrm{H} \\
650\end{array}$ & $\begin{array}{l}\text { Ank } \\
\text { ur } \\
3028\end{array}$ & $\begin{array}{l}\text { Biose } \\
\text { ed } \\
6588\end{array}$ & $\begin{array}{l}\text { MRC } \\
7017\end{array}$ & $\begin{array}{l}\mathrm{RC} \\
\mathrm{H} \\
650\end{array}$ & $\begin{array}{l}\text { Ankur } \\
3028\end{array}$ & $\begin{array}{l}\text { Bios } \\
\text { eed } \\
6588\end{array}$ \\
\hline $\mathbf{0}$ & - & - & - & - & - & - & - & - & - & - & - & - & - & - & - & - & - & - & - & - & - & - & - & - \\
\hline 1 & 30.1 & 21.1 & 16.5 & 25.7 & - & - & - & - & - & - & - & - & - & - & - & - & 4.0 & 5.1 & 3.4 & 4.1 & 7.9 & 7.3 & 9.9 & 11.0 \\
\hline 2 & 42.7 & 33.4 & 42.3 & 46.1 & 23.0 & 8.1 & 17.2 & 31.0 & 9.2 & 6.7 & 5.9 & 24.9 & 12.9 & 6.3 & 12.0 & 5.1 & 9.8 & 10.3 & 20.2 & 20.4 & 9.8 & 14.9 & 18.2 & 15.5 \\
\hline 3 & 54.5 & 44.4 & 57.2 & 56.7 & 42.3 & 42.7 & 28.5 & 45.6 & 21.5 & 13.6 & 16.0 & 38.3 & 19.9 & 10.7 & 16.7 & 10.6 & 25.6 & 20.0 & 27.4 & 24.8 & 26.1 & 24.9 & 25.6 & 26.5 \\
\hline 4 & 61.6 & 55.7 & 63.4 & 69.3 & 61.8 & 57.2 & 42.9 & 60.6 & 28.8 & 37.8 & 31.2 & 47.7 & 27.3 & 29.2 & 31.5 & 34.4 & 40.0 & 33.7 & 38.9 & 34.0 & 41.4 & 45.4 & 41.7 & 44.4 \\
\hline 5 & 78.0 & 63.9 & 83.3 & 74.5 & 63.5 & 62.1 & 58.2 & 70.7 & 46.6 & 53.6 & 39.6 & 70.7 & 37.3 & 37.7 & 43.2 & 50.6 & 51.9 & 47.8 & 55.1 & 38.6 & 50.2 & 51.8 & 47.7 & 52.4 \\
\hline 6 & 93.4 & 84.2 & 86.6 & 88.2 & 79.8 & 74.9 & 78.9 & 89.9 & 72.9 & 97.4 & 94.8 & 95.5 & 61.1 & 62.1 & 66.6 & 76.9 & 53.3 & 48.2 & 57.3 & 51.7 & 71.0 & 69.7 & 28.9 & 72.5 \\
\hline $\begin{array}{c}\text { Over all } \\
\text { average grade }\end{array}$ & 69.5 & 64.4 & 73.3 & 62.5 & 39.1 & 45.0 & 33.1 & 47.5 & 28.5 & 18.2 & 17.0 & 35.8 & 10.4 & 16.2 & 11.1 & 17.2 & 29.8 & 26.8 & 31.8 & 28.5 & 35.3 & 39.8 & 32.8 & 39.3 \\
\hline
\end{tabular}


Table.4 An overall reduction in the number of bolls and seed cotton yield per plant in corresponding to the different CLCuD grades, PDI and disease response (2012-2017)

\begin{tabular}{|c|c|c|c|c|c|c|c|c|c|c|c|c|c|}
\hline \multirow[t]{3}{*}{ Symptoms } & \multirow[t]{3}{*}{ DRS $^{\dagger}$} & \multicolumn{4}{|c|}{$\begin{array}{c}\text { Reduction \% over lowest } \\
\text { DRS }\end{array}$} & \multicolumn{2}{|c|}{$\begin{array}{l}\text { Overall } \\
\text { reduction } \\
(\%) \text { over } \\
\text { the lowest } \\
\text { DRS }\end{array}$} & \multicolumn{2}{|c|}{$\begin{array}{l}\text { Percent reduction } \\
\text { range in years where } \\
\text { "0" was recorded }\end{array}$} & \multicolumn{4}{|c|}{$\begin{array}{l}\text { Average reduction \% over } \\
\text { "0" DRS }\end{array}$} \\
\hline & & \multicolumn{2}{|c|}{ Faridkot } & \multicolumn{2}{|c|}{ Hisar } & \multirow[b]{2}{*}{$\begin{array}{l}\text { No. } \\
\text { bolls }\end{array}$} & \multirow[b]{2}{*}{ SCY } & \multirow[b]{2}{*}{ No. bolls } & \multirow[b]{2}{*}{ SCY } & \multicolumn{2}{|c|}{ Faridkot } & \multicolumn{2}{|c|}{ Hisar } \\
\hline & & bolls $\$$ & $\mathrm{SCY}^{\ddagger}$ & $\begin{array}{l}\text { No. } \\
\text { bolls }\end{array}$ & SCY & & & & & $\begin{array}{l}\text { No. } \\
\text { bolls }\end{array}$ & SCY & $\begin{array}{l}\text { No. } \\
\text { bolls }\end{array}$ & SCY \\
\hline $\begin{array}{l}\text { 0. The complete absence of } \\
\text { symptoms }\end{array}$ & 0 & - & - & & & 0.0 & 0.0 & 0 & 0 & 0 & 0 & 0 & 0 \\
\hline $\begin{array}{l}\text { 1. Symptoms of vein } \\
\text { thickening on a few upper } \\
\text { leaves }\end{array}$ & 1 & 11.1 & 12.2 & & & 11.1 & 12.2 & $1.5-32.2$ & $3.4-30.1$ & 13.6 & 15.0 & & \\
\hline $\begin{array}{l}\text { 2. Symptoms of vein } \\
\text { thickening, cupping and } \\
\text { curling on few upper leaves }\end{array}$ & 2 & 17.8 & 20.1 & 12.3 & 11.1 & 16.7 & 18.6 & $5.3-43.0$ & $9.8-46.1$ & 24.2 & 28.0 & 10.8 & 16.6 \\
\hline $\begin{array}{l}3.25 \% \text { plant affected with } \\
\text { vein thickening, cupping } \\
\text { and curling, leaf enations }\end{array}$ & 3 & 26.7 & 30.4 & 27.3 & 27.4 & 27.9 & 30.0 & $13.7-60.2$ & $20.0-57.2$ & 33.9 & 38.0 & 33.8 & 39.3 \\
\hline $\begin{array}{l}\text { 4.50\% plant affected with } \\
\text { vein thickening, cupping } \\
\text { and curling, leaf enations }\end{array}$ & 4 & 37.4 & 41.3 & 42.5 & 44.4 & 41.3 & 44.3 & $24.5-65.1$ & $33.7-69.3$ & 41.0 & 46.7 & 53.9 & 57.6 \\
\hline $\begin{array}{l}5.75 \% \text { plant affected } 5 \text { with } \\
\text { vein thickening, cupping } \\
\text { and curling, leafy enation }\end{array}$ & 5 & 49.6 & 53.0 & 53.6 & 54.3 & 52.9 & 55.3 & $37.5-76.0$ & $38.6-83.3$ & 51.4 & 56.1 & 62.1 & 59.6 \\
\hline $\begin{array}{l}\text { 6. Plants stunted severely and } \\
\text { complete plant affected } \\
\text { with vein thickening, } \\
\text { cupping and curling and } \\
\text { leafy enation }\end{array}$ & 6 & 69.9 & 73.5 & 67.7 & 71.8 & 64.9 & 68.4 & $41.0-89.7$ & $48.2-93.4$ & 64.7 & 70.9 & 74.5 & 79.6 \\
\hline
\end{tabular}

$\dagger$ : disease rating scale, $\ddagger$ : seed cotton yield, $\S$ number of bolls per plant 
Table.5 An overall reduction in the number of bolls and seed cotton yield per plant in corresponding to the different CLCuD grades, DSI and midpoint value (2012-2017)

\begin{tabular}{|c|c|c|c|c|c|c|c|c|c|c|c|c|}
\hline \multirow[t]{2}{*}{ Symptoms } & \multirow[t]{2}{*}{ DRS $^{\dagger}$} & \multirow{2}{*}{$\begin{array}{c}\text { DSI }^{\mathrm{gI}}(\%) \\
\text { correspon } \\
\text { ding to } \\
\text { DRS }\end{array}$} & \multicolumn{2}{|c|}{$\begin{array}{l}\text { Pooled reduction } \\
\text { (\%) over "0" } \\
\text { DRS }\end{array}$} & \multirow[t]{2}{*}{$\begin{array}{l}\text { Disease } \\
\text { response }\end{array}$} & \multicolumn{3}{|c|}{ Mathematical values* } & \multirow{2}{*}{$\begin{array}{c}\text { Adopted } \\
\text { Disease } \\
\text { Severity } \\
\text { Index (\%) }\end{array}$} & \multirow{2}{*}{$\begin{array}{c}\text { Midpoint } \\
\text { value for } \\
\text { adopted } \\
\text { DSI }\end{array}$} & \multirow{2}{*}{$\begin{array}{c}\text { Proposed } \\
\text { Disease } \\
\text { index } \\
(\%)\end{array}$} & \multirow{2}{*}{$\begin{array}{c}\text { Midpoin } \\
\text { t value } \\
\text { for } \\
\text { proposed } \\
\text { DSI }\end{array}$} \\
\hline & & & $\begin{array}{c}\text { No. } \\
\text { bolls }\end{array}$ & $\mathrm{SCY}^{+}$ & & $\mathrm{LCL}$ & UCL & $\begin{array}{c}\text { Midp } \\
\text { oint }\end{array}$ & & & & \\
\hline o. Complete absence of symptoms & 0 & 0 & 0.0 & 0.0 & I & 0 & 0 & 0 & 0 & 0 & 0 & \\
\hline $\begin{array}{l}\text { 1. Symptoms of vein thickening on } \\
\text { a few upper leaves }\end{array}$ & 1 & 16.7 & 13.6 & 15.0 & HR & 0.1 & 16.5 & 8.4 & $0.1-10$ & 5.05 & $0.1-15.0$ & 7.6 \\
\hline $\begin{array}{l}\text { 2. Symptoms of vein thickening, } \\
\text { cupping and curling on few } \\
\text { upper leaves }\end{array}$ & 2 & 33.3 & 24.2 & 28.0 & $\mathrm{R}$ & 16.6 & 33.2 & 25.0 & $11-20$ & 15.5 & $15.1-30.0$ & 22.6 \\
\hline $\begin{array}{l}\text { 3. } 25 \% \text { plant affected with vein } \\
\text { thickening, cupping and curling, } \\
\text { leaf enations }\end{array}$ & 3 & 50.0 & 34.6 & 38.6 & MR & 33.3 & 49.9 & 41.7 & $21-30$ & 25.5 & $30.1-45.0$ & 37.6 \\
\hline $\begin{array}{l}\text { 4. } 50 \% \text { plant affected with vein } \\
\text { thickening, cupping and curling, } \\
\text { leaf enations }\end{array}$ & 4 & 66.7 & 43.3 & 49.1 & MS & 50.0 & 66.6 & 58.4 & $31-40$ & 35.5 & $45.1-55.0$ & 50.1 \\
\hline $\begin{array}{l}\text { 5. } 75 \% \text { plant affected } 5 \text { with vein } \\
\text { thickening, cupping and curling, } \\
\text { leafy enation }\end{array}$ & 5 & 83.3 & 54.1 & 59.1 & S & 66.7 & 83.3 & 75.1 & $41-50$ & 45.5 & $55.1-65.0$ & 60.1 \\
\hline $\begin{array}{l}\text { 6. Plants stunted severely and } \\
\text { complete plant affected with vein } \\
\text { thickening, cupping and curling } \\
\text { and leafy enation }\end{array}$ & 6 & 100 & 65.9 & 72.1 & HS & 83.4 & 100 & 91.8 & $51<$ & 75.5 & $65<$ & 82.6 \\
\hline & & & & & & & & & & & & \\
\hline
\end{tabular}

$\dagger:$ disease rating scale, $¥$ : seed cotton yield, $\S$ number of bolls per plant, $\uparrow$ : disease severity index

*Midpoint rule or so-called trapezoidal integration method (Campbell and Madden, 1990);

${ }^{\circledR} \mathrm{I}=$ immune, $\mathrm{HR}=$ highly resistant, $\mathrm{R}=$ resistant, $\mathrm{MR}=$ moderately resistant, $\mathrm{MS}=$ moderately susceptible, $\mathrm{S}=$ susceptible, HS= highly susceptible 
Fig.1 Scattered diagram for regression analysis (a) SCY $=0.4748+1.0321 \times$ No. bolls (b) SCY $=-2.0426+0.6923 \times$ PDI
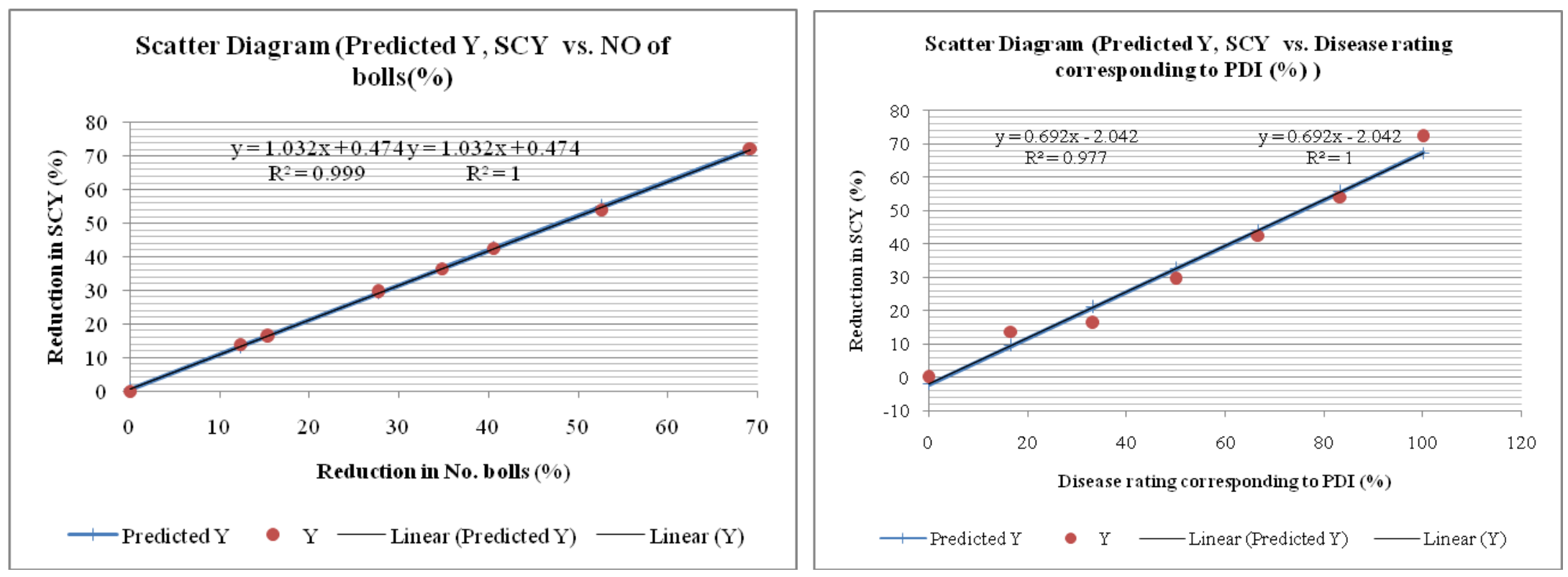
Figure.2 Dynamics of CLCuD rating scales on reduction in the number of bolls (NB) and seed cotton yield (SCY) ( $\mathrm{g}$ plant $\left.{ }^{-1}\right)$ in different Bt cotton varieties at all the locations (pooled mean) corresponding to the PDI/DSI analogous to rating scale, mathematical midpoint value of DRS, DSI and proposed disease response during 2012-2017

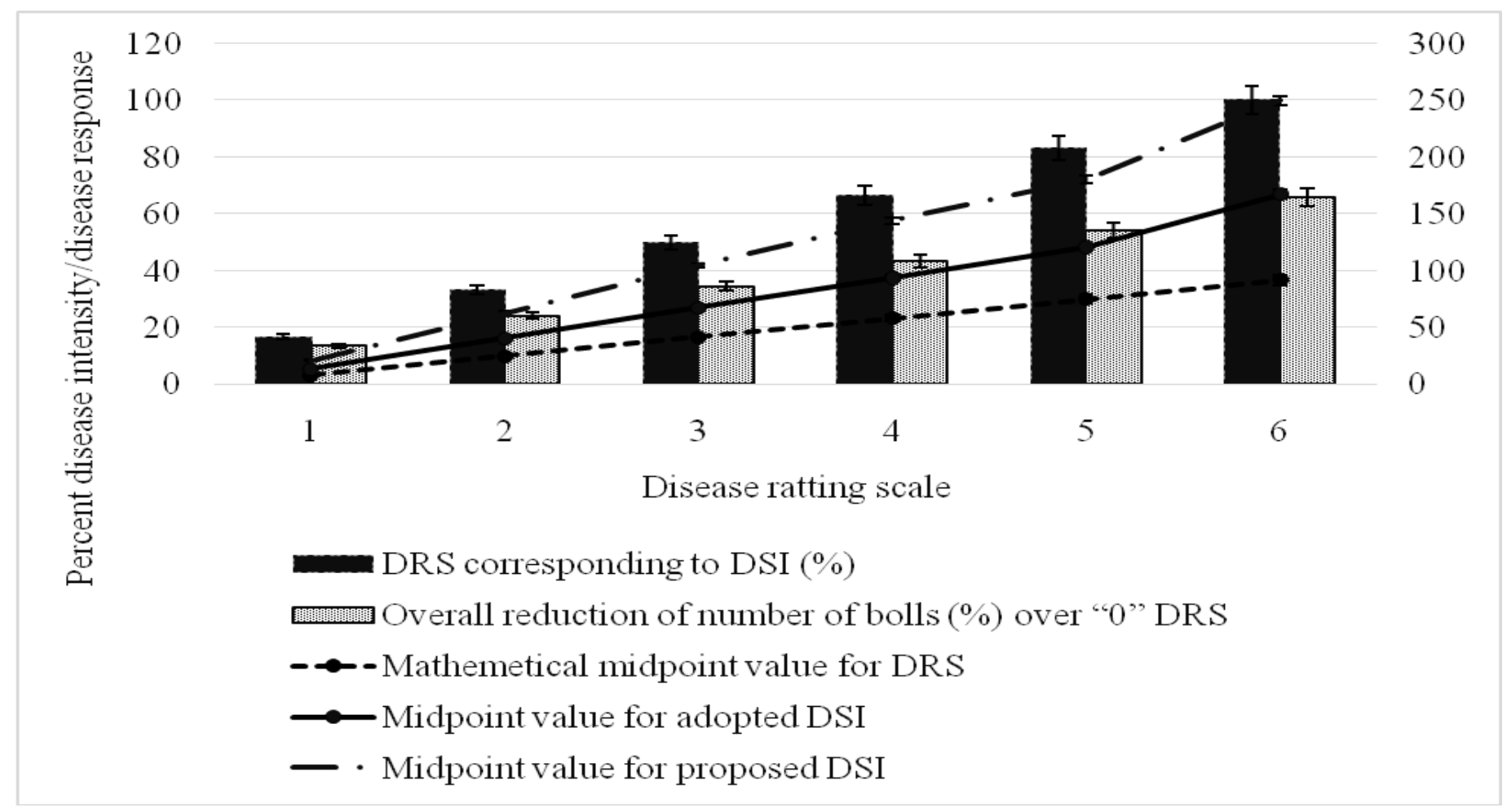


The nucleotide sequence analysis of the CLCuD shared the highest level of nucleotide sequence identity (98\%) with Cotton leaf curl Multan virus (CLCuMuV) during 2014 and 2015 (Qadir et al., 2019) and CLCuMuVRajasthan strain (97\% identity) during 2012 to 2014 (Biswas et al., 2020). Also, as reported earlier that whitefly cryptic species Asia II-1 has mostly occurred in the cottongrowing areas of northwest India and during this study, the whitefly populations were confirmed as whitefly cryptic species Asia II 1 based on the mtCOI sequence analysis (Biswas et al., 2020). These results are from other collaborative studies (Qadir et al., 2019; Biswas et al., 2020).

During the study period, A 0-6 disease rating scale described by Akhtar et al., (2010) has been modified and used in the present studies. As in previous studies, the disease rating scale that has been generally adopted is based on percent disease intensity (PDI \%). The formula used to calculate PDI has been reported by McKinney (1923). According to this formula, individual symptomatic plant rating for each genotype are summed up and divided by the number of infected plants to calculate the average grade. The corresponding PDI was calculated using the average grade divided by total rating scale i.e. 6 and multiplied by 100 .

The formula to describe disease reaction/response (DSI) by Akhtar et al (2002, 2010) $(0.1-10=\mathrm{HR}, 10.1-20.0=\mathrm{R}, 21-30=\mathrm{MR}$, $31-40=\mathrm{MS}, 41-50=\mathrm{S},>50=\mathrm{HS}$ ) was found to be quite arbitrary as no scientific basis for arriving at it was presented. However, in the present studies the yield reduction vis-à-vis grades 1-6 has been presented. If all plants fall under DRS (symptom rating) "1" which is corresponding to highly resistant type symptom (DRS 1) in a particular variety/hybrid/germplasm the corresponding PDI (\%) will be $16.7 \%$ (mathematical calculation), as the disease severity index has been characterized under six-point scale, each point is thus separated by $100 / 6$ i.e. $16.7 \%$ with yield loss about 13.6-15\% (Table 4) as per our findings. According to the previously adopted disease response scale, this " 1 " rating or disease reaction is highly resistant which equals to $16.7 \%$ PDI falls under the previously described disease response category - 10.1-20 disease severity index $10.1-20 \%$ which is equal to the resistant response category described by of Akhtar et. al. (2010). Similarly, this situation occurs with the other DRS corresponding to the previously adopted PDI reaction response. In the case of the " 6 " grade of rating scale or disease reaction HS which equals $100 \%$ PDI, it equals > 50\% which is arbitrarily and without any scientific yield loss data or mathematical calculation. However, if we look at yield reduction in the case of grade six in our studies it ranges between $65.9-72.1 \%$ (Table 4).

The study conducted from 2012 to 2017, we found that the pooled reduction in the number of bolls and seed cotton yield over healthy plants (0 DRS) corresponding to DRS 1, 2, 3, 4, 5, 6 were in a similar trend. The pooled reduction in seed cotton yield over healthy plants (0 DRS) corresponding to DRS 1, 2, 3, 4, 5, 6 were $15,28,38.6,49.1,59.1$ and $72.1 \%$, respectively (Table 4). As per the mathematical calculation, the disease rating scale 1, 2, 3, 4, 5, 6 corresponding to DSI and disease response is $16.3(\mathrm{HR}), 33.3(\mathrm{R}), 50$ (MR), 66.7 (MS), 83.3 (S) and $100 \%$ (HS), respectively. Also, as per the midpoint rule or so-called trapezoidal integration method (Campbell and Madden, 1990) the midpoint value corresponding DRS 1, 2, 3, 4, 5, 6 should be 8.4, 25, 41.7, 58.4, 75.1 and 91.8, respectively. Statistically, the midpoint value for the previously adopted disease severity index looks very low then the actual i.e. 5.05, $15.5,25.5,35.5,45.5$, and 75 , respectively. 
However, the midpoint value for the proposed DSI for disease response corresponding to DRS comes to 7.6, 22.6, 37.6, 50.1, 60.1 and 82.6 , respectively and is relatively nearer to the statistical midpoint value for the corresponding DSI and DRS. Also, the pooled reduction in seed cotton yield over diseasefree plants (0 DRS) is almost nearer to the proposed DSI and its midpoint value corresponding DRS 1, 2, 3, 4, 5, 6 (Table 4 and 5).

The study showed that the percent reduction in seed cotton yield is more or less similar to the reduction in the number of bolls corresponding to the symptom/disease rating scales (DRS) and percent disease intensity (PDI). Similarly, the scattered diagram of the regression prediction model for seed cotton yield and the number of bolls per plant was recorded to be $\mathrm{SCY}=0.4748+1.0321 \mathrm{x}$ no. bolls per plant $\left(\mathrm{R}^{2}=0.0999\right)$ (Fig.1a). The scattered diagram of the regression prediction model for seed cotton yield and DRS was recorded to be $\mathrm{SCY}=-2.0426+0.6923 \times \mathrm{DRS}$ $\left(\mathrm{R}^{2}=0.9777\right)$ (Fig. 1b).

When we compare between the previously adopted PDI corresponding to different reaction grades/scales $0.1-10=\mathrm{HR}, 10.1$ $20.0=\mathrm{R}, \quad 21-30=\mathrm{MR}, \quad 31-40=\mathrm{MS}, \quad 41-50=\mathrm{S}$, $>50=\mathrm{HS}$, it is towards the lower side compared to the corresponding losses under different disease ratting scale/grades. Figure 2 shows the pooled reduction in seed cotton yield and the number of bolls over the years and mid-point values for old disease response scale, mathematical PDI, and proposed disease response scale. The line graphs of three midpoint values are in alignment with the reduction in the SCY and the number of bolls per plant. The mathematical midpoint value is higher than the proposed disease response scale while the midpoint value for the old disease response scale is very lower. However, the midpoint value for the proposed disease response scale is far-far better in terms of alignment with the yield loss is proportionate to the PDI and hence may prove to be the best to determine the resistance response of a particular genotype for future breeding improvement programmes. Thus, the previously adopted/reported scale used for resistant material screening seems to be arbitrary and is far-far lower than the new proposed scale and even the midpoint value of actual (Fig. 2, Table 5).

It is proposed that the proposed reaction/response scale would be much more justifiable and reliable for decision making for determining the level of resistance or susceptibility in the genotypes, because the estimated severity otherwise may be much higher than the actual severity. As the yield loss is in proportionate to the old resistance response scale may not occur, and the lines/germplasm with good yield potential may be ignored for future breeding improvement programmes. Hence, we propose that a midpoint value-based new resistance response scale may be utilized for decision making in future resistance screening programmes to enhance cotton production and productivity.

\section{Acknowledgment}

The author acknowledges the support from Director, ICAR-Central Institute for Cotton Research, Nagpur, and Project Coordinator All India Coordinated Research Project on Cotton for providing necessary facilities and fundings for conducting the experiments.

Conflict of Interest Statement: No potential conflict of interest was reported by the author(s).

\section{References}

Ahmed, Z. (1999) Prospects and bottlenecks of cotton crop in Pakistan. The Pak Cotton Grower 3:6-7 
Akhtar, K.P., Haider, S., Khan, M.K.R., Ahmad, M., Sarwar, N., Murtaza, M.A.\& Aslam, M. (2010). Evaluation of Gossypium species for resistance to leaf curl Burewala virus. Ann Appl Biol 157:135-147.

Akhtar KP, Khan MSI (2002) Modified scale for the assessment of cotton leaf curl virus $(\mathrm{CLCuV})$. Pakistan J Phytopath 14:88-90.

Ali M, Ahmed Z, Tanveer M, Mahmood T (1995) Cotton leaf curl virus in the Punjab. Current Situation and review of work p 47.

Anonymous (2019) All India Coordinated Research Project on Cotton-Project Coordinator's Report. 2018-19.

Anonymous (2017)

https://www.ibef.org/exports/cottonindustry-india.aspx

Biswas KK, Bhattacharyya UK, Palchoudhury S, Balram N, Kumar A, Arora R, Sain SK, Kumar P, Khetarpal RK, Sanyal A, Mandal PK (2020) Dominance of recombinant cotton leaf curl MultanRajasthan virus associated with cotton leaf curl disease outbreak in northwest India. PLoS ONE 15(4), e0231886. https://doi.org/10.1371/journal.pone.0231 886

Briddon RW (2003) Cotton leaf curl disease, a multicomponent begomovirus complex. Mole Pl Patho 4:427-434.

Briddon RW, Mansoor S, Bedford ID, Pinner MS, Markham PG (2000) Clones of cotton leaf curl geminivirus induce symptoms a typical of cotton leaf curl disease. Virus Genes 20:17-24.

Brown JK, Zerbini FM, Navas-Castillo J, Moriones E, Ramos-Sobrinho R, Silva JC, Fiallo-Olivé E, Briddon RW, Hernández-Zepeda C, Idris A, Malathi VG, Martin DP, Rivera-Bustamante R, Ueda S, Varsani A (2015) Revision of Begomovirus taxonomy based on pairwise sequence comparisons. Arch Virol 160:1593-1619.

Campbell CL, Madden LV (1990) Introduction to plant disease epidemiology. John
Wiley, Sons, New York.

Farooq A, Farooq J, Mahmood A, Batool A, Rehman A, Shakeel A, et al., (2011) A review of cotton leaf curl virus disease $(\mathrm{CLCuD})$ as a serious threat to cotton productivity. Australian J Crop Sci 5:1823-1831.

Idris AM (1990) Cotton leaf curl disease in Sudan. Med. Fac. Land bow, Rijksunir, Gent 55:263-267.

Kumar A, Snehi, SK, Raj SK, Kumar J, Khan JA (2011) Association of Cotton leaf curl Burewala virus and its satellite molecules with leaf distortion symptoms of cotton in India. New Dis Rep 24:18.

Kumar A, Kumar J, Khan ZA, Yadav N, Sinha V, Bhatnagar D, Khan JA (2010) Study of beta satellite molecule from leaf curl disease of sunn hemp (Crotalaria juncea) in India. Virus Genes 41:432-440.

McKinney $\mathrm{HH}$ (1923) Influence of soil temperature and moisture on infection of wheat seedling by Helminthosporium sativum. J Agri Res 26:195- 217.

Mahmood T, Tahir M, Tanveer M, Mirza MB (1996) Effect of cotton leaf curl virus on yield components and fiber properties of four commercial varieties. Pakistan J Phytopath 8:68-70.

Mansoor S, Amin I, Briddon RW (2008) Cotton leaf curl disease. In: Mahy BWJ, Van Regenmortel MHV (Eds) Encyclopedia of Virology, 5 pp 563-569 Oxford: Elsevier Publisher

Monga D (2014) Status of cotton leaf curls virus disease in India. In: 6th meeting of Asian Cotton Research and Development Network. Dhaka, Bangladesh. 18-20 June 2014, DOI: www.icac.org.

Monga D, Chakrabarty PK, Kranthi KR (2011) Cotton leaf curl disease in India-recent status and management strategies. Presented in the $5^{\text {th }}$ meeting of the Asian Cotton Research and Development Network. Lahore, Feb. 23-25 DOI: at www.icac.org.

Monga D, Narula AM, Raj S (2001) Management of cotton leaf curl virus- A dreaded disease in north India. National 
seminar on Sustainable cotton production to meet the future requirement of the industry. Kapas Vikas Nideshalya, Directorate of cotton development, Government of India. pp112-115.

Monga D, Raj S, Mayee CD (2004) Strategies for cotton leaf curl virus disease management. In: Changing World-OrderCotton Research, Development and Policy in Context. ANGRAU, Hyderabad, August 10-12, In: Chauhan MS, Saini RK (Eds) Recent Advances in Cotton Research and Development. P 205-203.

Monga D, Raj S, Varma SK (1998) Preliminary investigations on reaction of cotton germplasm to leaf curl and losses caused by the disease. National Symposium on "Present Scenario in diseases of Oilseeds and Pulses" Aurangabad. February 17-19.

Monga D, Sekhon PS, Beniwal J, Singh D, Patil PV, Dhoke PK, Ingole OV, Perane RR, Chattannavar SN, Sree Lakshmi B, Rao MSL (2012) Avoidable losses due to cotton diseases in India. In: Siwach SS, Sangwan RS, Chauhan MS, Sangwan O (Eds) Global Cotton Production Technologies vis-a-vis Climate Change. Cotton Research and Development Association, CCS HaryanaAgriculturaI University, Hisar - 125 004, India. pp 6267.

Narula AM, Monga D, Chauhan MS, Raj S (1999) Cotton leaf curls virus disease in
India-The Challenge ahead. J Cotton Res Develop 13:129-38.

Nateshan HM, Muniyappa V, Swanson MM, Harrison BD (1996) Host range, vector relations and serological relationships of cotton leaf curl virus in South India. Ann Appl Biol 126:233-244.

Qadir R, Khan ZA, Monga D, Khan JA (2019) Diversity and recombination analysis of Cotton leaf curl Multan virus: a highly emerging begomovirus in northern India. BMC Genomics 20, 274 https://doi.org/10.1186/s12864-019-56402

Sattar MN, Iqbal Z, Tahir MN, Ullah S (2017) The prediction of a new CLCuD epidemic in the old world. Frontier Microbio 8:631, https://doi.org/10.3389/fmicb.2017.00631

Singh D, Gill JS, Gumber RK, Singh R, Singh S (2013) Yield and fibre quality associated with cotton leaf curl disease of Bt-cotton in Punjab. J Environ Biol 34:113-6.

Singh J, Sohi AS, Mann HS, Kapoor SP (1994) Studies on whitefly Bemisia tabaci (Genn.) transmitted cotton leaf curl virus disease in Punjab. J Insect Sci 7:194-198.

Zerbini FM, Briddon RW, Idris A, Martin DP, Moriones, E, Navas-Castillo J, RiveraBustamante R, Roumagnac P, Varsani A (2017) ICTV Virus Taxonomy Profile: Geminiviridae. J Gen Virol 98:131-133.

\section{How to cite this article:}

Satish Kumar Sain, Dilip Monga, Man Mohan, Aman Sharma and Jagdish Beniwal. 2020. Reduction in Seed Cotton Yield Corresponding with Symptom Severity Grades of Cotton Leaf Curl Disease (CLCuD) in Upland Cotton (Gossypium hirsutum L.). Int.J.Curr.Microbiol.App.Sci. 9(11): 3063-3076. doi: https://doi.org/10.20546/ijcmas.2020.911.372 